\title{
Proposition of Ergonomic Guidelines to Improve Usability of PLM Systems Interfaces
}

\author{
Perrine de Pinel ${ }^{1}$, Nicolas Maranzana ${ }^{1}$, Frédéric Segonds ${ }^{1}$, \\ Sylvain Leroux ${ }^{2}$, and Vincent Frerebeau ${ }^{3}$ \\ ${ }^{1}$ Arts et Métiers ParisTech, LCPI, \\ 151 bd de l'Hôpital, 75013, PARIS, France \\ ${ }^{2}$ NextIS-Consulting, \\ 18 rue Mogador, 75009 PARIS, France \\ ${ }^{3}$ Vincent FREREBEAU \\ Independent consultant \\ p.depinel@gmail.com
}

\begin{abstract}
Evolutions of PLM systems are mainly directed towards the integration of more lifecycles stages to offer more PLM functions and information systems. At first, PLM were developed and intended only for engineers in detailed design phases. As engineers are familiar with complex and sparse interfaces, PLM software are not really ergonomic. The emerging problem in the past few years is the lack of evolution of PLM software, decreasing significantly its efficiency and utilization. But now, PLM integrate entire lifecycle of the product, so interfaces should be readjusted so that every actor, other than engineers, can easily use it.

In this paper, we assume that simplify and dynamize PLM software interfaces, by meeting usability features and user experience, make the system more interactive and coherent. In a first time, two states of art are realized. The first is about PLM evolution and web interfaces, the second about user experience and usability. A combination between agile method and user-centred design method are applied to these states of art in order to generate PLM software prototypes. Finally, user tests will be conducted on these prototypes in an incrementally and iterative way to correct and to validate them. The final target is to propose some ergonomic guidelines in order to create a generic interface adapted to several kinds of PLM software.
\end{abstract}

Keywords: Ergonomic, PLM, usability, user experience, agile method.

\section{Introduction PLM and Challenge}

In the earlier 2000s, PLM emerged as a solution to adapt industrial design to the demands of globalization. Indeed, as PLM addresses the entire lifecycle of the product, it has a cross-functional nature and deals closely with the way a company runs (Garetti et al., 2005).

For Amann (2002), over the past several years, PLM has emerged as a term to describe a business approach for the creation, management and use of productassociated intellectual capital and information throughout the product lifecycle. 
According to Pernelle and Lefebvre (2006), with the increasing concentration of editors and the obsession of lowering information technology costs, too few companies are aware that PLM system design has to evolve to follow user's expectations. Indeed, generic PLM methods are not adapted to the actual PDM platforms configuration causing some heterogeneity issues in the system. Other difficulties found in the PLM system design phase are the complexity and the lack of flexibility in the product models. Pernelle and Lefebvre (2006). This can be linked to the level of details of some standards (ISO 10303-1, 1994), which affects the data flowing fluidity.

These different points show the need to act on the PLM design and on its evolution through time. We intend to act along three main lines of PLM: product, processes and organization. We attempt to prove that adding innovation in PLM software design by including ergonomic and usability features can enhance user satisfaction.

\section{State of Art}

This state of art explains how an ergonomic approach can be integrate during the process PLM. It traces and explain the different rules attempting to improve process and interface PLM in the aim to satisfy the user.

\subsection{Introduction of Concurrent Engineering and Ergonomic Approach}

Introduction of concurrent engineering implies modifying design habits and requires changes in the project structure. This methodology involves different dimensions such as organizational, social, technical and economic (Bossard, 1997). In this context, it is possible to think that ergonomics can take place in this process at several levels (Fadier, 1997; Sagot, 1999).

The design process is complex and includes different cultures, actors and methods. Also, collaboration can be difficult and tensions may appear (Gronier, Sagot, 2005). A good collaboration environment requires some infrastructures such as databases, processes, and applications. This leads the company towards a common model oriented in an intuitive way that can be consulted by PLM users. With this kind of process, decisions can become more affordable and more understandable. Ergonomic approach can be considered as part of resource information and so assists the development process.

According to Kim et al. (2008), ergonomic analyses are not well managed in PLM. Indeed, they notice that the effective management of human information, along with concurrent ergonomic analysis is very important for each cycle of the product engineering in PLM. As stated Segonds et al. (2011) and Maranzana et al. (2011), PLM software evolution is still developed with a lack of maturity, of consistency, of relationship between modules.

According to Sagot (1999), integrating human factors in the design product today is an important point. Developing a multidisciplinary approach with engineering and social sciences, as ergonomics, is a huge challenge and a new way of thinking and collaborating. Nowadays, innovating in product design, it is important to meet users' needs and expectations through ergonomics. 
The aim of ergonomics is to understand interactions between people and systems, define constraints of the user activities and determine the list of tasks PLM users will have to manage. Analyze the users' needs is an important first part of the approach aiming at reducing the problem scope. Therefore requirements depend on use and esteem needs. In fact, changing only one demand can have a significant impact on the system. Recommendations will help to define some guidelines to redefine the systems. They are active on the visible aspect of the interface and contribute to improve the content of the dialogue between the user and the system.

\subsubsection{Ergonomics Standards}

Standards are a set of rules to design and develop systems by ensuring a high level of comfort, performance, satisfaction and security when using a system. Two types exist: management process standards and standards specific to the product. The first type contains one of the most famous standards of the user-centered part design cycle, (ISO 13407, 1999). It's a guide with source information and user-centered organization principles such as: planning and management of design methods, technical aspects of human factors, usability features and general ergonomic principles. In the second type, designers can find some recommendations and the different manners of interacting when designing interfaces. For example, ISO 924110 (1993), the dialogue (adaptation to the task, ease of use, error tolerance...); ISO9241-13 (1998): the guidance system, etc. Usability features is an important part of the ergonomic approach and a reference in an expert evaluation of interfaces.

\subsection{Human Computer Interaction and Usability Features}

As stated by Juristo et al. (2007), the field of human computer interaction is often related to the usability of software systems. The authors developed a state of art in order to provide some recommendations on how to improve software system usability. Several authors refer to such recommendations named differently: design heuristics (Nielsen, 1993), usability rules (Shneiderman, 1998), usability principles (Preece et al., 1994; Constantine and Lockwood, 1999), ergonomics principles (Scapin and Bastien, 1997), usability patterns (Tidwell, 2005; Welie, 2003), etc.

According to Juristo et al., (2007), software usability is a quality attribute listed in a number of classifications (ISO 9126, 2001; Boehm, 1978). Nielsen (1993) clarifies a standard definition of usability as learnability and memorability of software system, its efficiency of use, ability to avoid and manage user errors and user satisfaction. ISO 9241-11 (1998) defines usability as "the extent to which a product can be used by specified users to achieve specified goals with effectiveness, efficiency and satisfaction in a specific context of use'. In short, usability is also generally referred to as "quality in use", (ISO 14598, 1999). Many studies extol the virtues and benefits of usability (Trenner, 1998; Battey, 1999; Donahue, 2001; Griffith, 2002; Black, 2002): it improves productivity and raises team moral, reduces training and documentation costs, improves user productivity, increases e-commerce potential, etc. (Juristo et al., 2007). Usability is still insufficiently considered in most software systems (Seffah and Metzker, 2004). 
Since 1990, usability inspection is the new way to evaluate user interface. According to Nielsen (1993), there are four basic ways of evaluating it: automatically (usability measured by running interface test programs), empirically (tests the interface with real users), formally (use precise models to calculate usability) and informally (based on experience of the evaluators). This kind of inspection leads us to have a large vision on the different aspects of PLM software evaluation.

Software usability is defined as an approach related to design, evaluation and use of human computer interaction, in order to optimize the compatibility between user, activity and software interfaces. Usability allows avoiding issues that could happen and tend to ensure a high performance and an ease of use. To resume, the aim is to design, correct and transform devices and software, by adapting them to human capabilities. It is important to preserve physic, mental and social aspects, preventing negative consequences during the user activities.

According to Brangier et al., (2009), scientific input of software usability can be defined in three types. Firstly, software usability defines some recommendations about style and content presented during interaction.

The goal is to guide how to design, organize and specify user's activities, taking into account human factors within the technology. Establish some rules based on experiments, methods and practices whose efficiency and effectiveness are verified. Secondly, software usability tries to specify some models of human computer interaction. It defines ways how the end users should interact with the machine and tries to identify cognitive process developed by them. It elaborates a cognitive and interactive functioning model of the user linked to the machine. Thirdly, software usability takes part of the process and methods development ensuring sensorial and cognitive compatibility and user acceptance optimizing usability features.

The aim of these three approaches is to reduce the distance between user and interfaces and to promote the user acceptance.

\subsection{User's Acceptability of the System}

Technological acceptability defines how users find a way to take control of a given system. It is based on how he deals and reacts to issues linked to the new technologies. To check compatibility between technical and psycho-physiologic features of the user can be interesting. For example, Shneiderman (1980) worked on compatibility between software features with the user operating mode regarding the ease of use and usability features. According to Nielsen (1993), acceptability is decomposed in many levels (Fig.1).

To design and evaluate interfaces, it can be useful to apply knowledge on cognitive, operational and social functioning.

These different ergonomic rules can be adapted to PLM software. In order to apply these recommendations in this kind of interfaces, we can use an agile software development since this approach is widely used in design engineering environments. It involves and takes into consideration the user and the client so as to adapt the development process. 


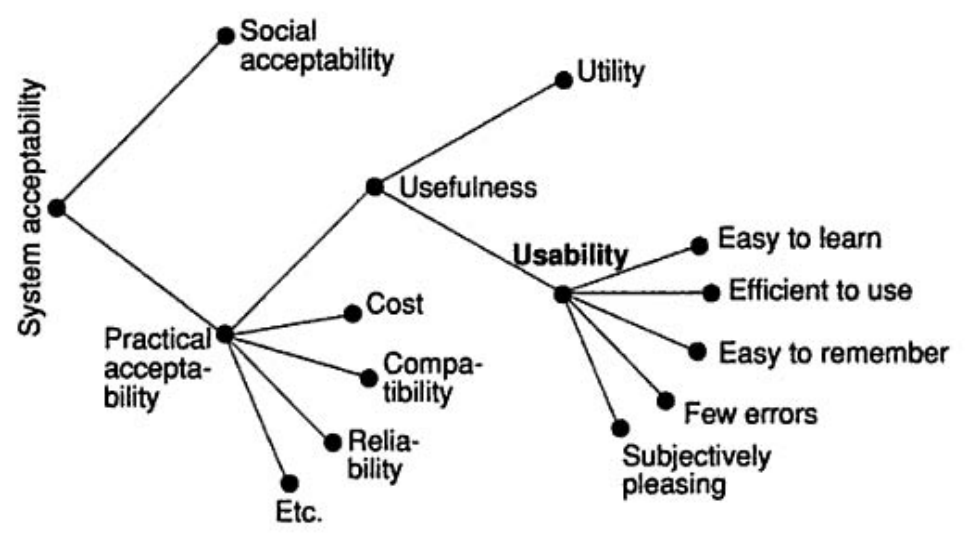

Fig. 1. System acceptability model adapted from Nielsen (1993)

\section{Proposition of PLM Software Interfaces Improvement through User Centered Method and Agile Development}

Losada et al., (2012) propose a guide to agile development of interactive software with a user centered driven methodology. They propose to improve the product usability by applying the maximum ergonomic recommendations and standards as possible. Even if they are different way of interaction, combining agile method with user-centered design could be a compelling approach to improve the efficiency of a PLM system. The aim is to understand the needs and tasks in order to adapt development principles by fostering individuals and interaction over process and tools and customer collaboration over contract negotiation.

Agile method objective is used to develop software in an incremental and iterative way. The main advantage is its flexibility during the development. This method is oriented on code development and ensures an adapted implementation of the functionalities. It tends to improve how software and process are developed. On the other hand, user centered design method is focused on the interaction in which the user will participate and to register the user's opinion about the application. User experience identify the expectations and cognitive brakes; study the expectations and needs; analyze user perception and analyze performance (Garett, 2002). It improve productivity and efficiency of the company by saving time, simplifying actions, and adding specific features. Both methods allow matching the users' requirement and their satisfaction while using the software.

Segonds (2011), developed a generic model based on a collaborative environment by integrating an agile development method. We base our approach on the same process by proposing a new model which describe how integrate user experience at each stage of the PLM development on the agile method. (Fig.2).We can propose the following hypothesis: Integrating User Experience and some Best Practices ergonomic during project development, facilitates the acceptance of users in the use of PLM in the company. 


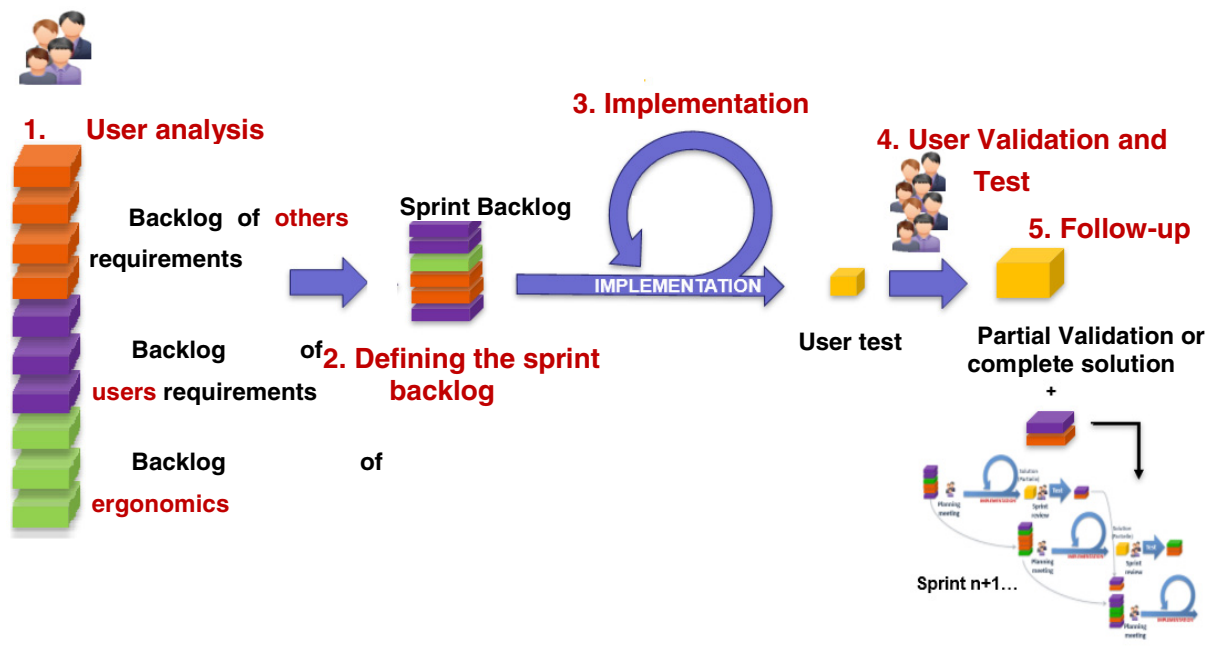

Fig. 2. PLM interface model design using a user centred method combined with agile development, adapted from Segonds and NextIS, (2011)

\section{$4 \quad$ Experimental Plan}

This protocol is a proposition; our hypothesis are not tested yet. Protocols below are the process we will used to answer them.

\subsection{Integration of Best Practices Ergonomic User Centred}

The integration of user experience in the agile model is represented by 5 steps.

\section{User analysis}

- To get acquainted with the project, to take account vision and objectives of the Product Owner

- Analyze the current situation, user behavior

- Define the target users and their characteristics (representative sample)

- Ensure user awareness to usability

- Identification requirements = Interview

2. Defining the sprint backlog

- Build Personas from the characteristics of users (Grudin \& al. 2002)

- Write scenarios

- Design a navigation scheme based on scenarios

- Write detailed uses cases

- Prioritize uses cases with the Product Owner (P.O)

\section{Implementation}

- Describe the guidelines and standards for interface design

- For each use case, design a Digital Mock-Up 
- Validate the mock-up developed, with users (Feedback)

- Achieve the development of the mock-up for the iteration (developers)

4. User Validation and Test

- Submit to the P.O the work during the iteration

- Validate the software developed with users

\section{Follow-up}

- Follow-up audit

- Change management

In order to validate our hypothesis, we establish a user test.

\subsection{User Test}

According to Nielsen and Laundauer (1993), 5 users can detect $75 \%$ of usability problems on an interface (Fig 3.). Each of the five users made two scenarios, one without ergonomic requirements and one with them. At the end, we compare the objective and subjective measures in order to check the productivity gain achieved with the ergonomic requirements. (Table 1).

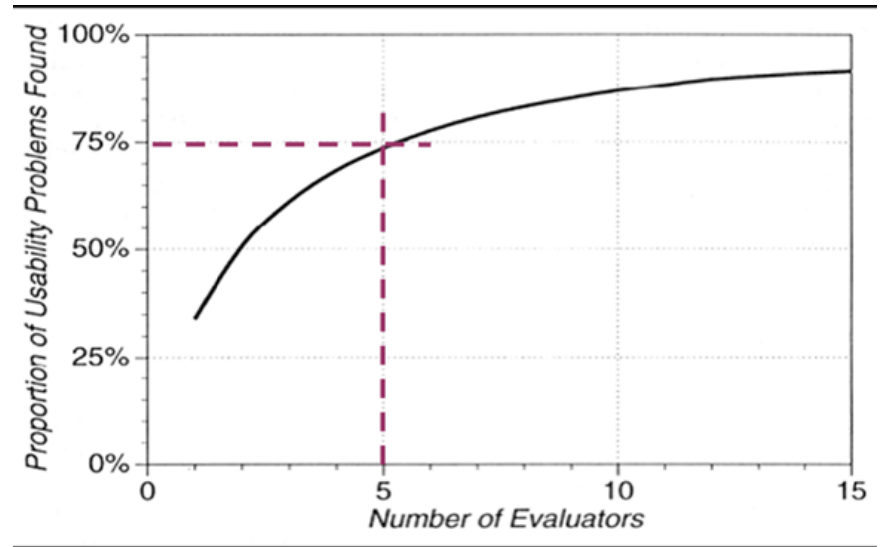

Fig. 3. Graphical representation adapted from Nielsen and Laundauer (1993) 
Table 1. Table allowing to compare a scenario before and after the integration of ergonomic requirements in order to obtain objective and subjective measures

\begin{tabular}{|c|c|c|c|c|c|}
\hline & $\begin{array}{c}\text { Time to } \\
\text { complete } \\
\text { the } \\
\text { scenario }\end{array}$ & $\begin{array}{c}\text { The } \\
\text { number of } \\
\text { mouse } \\
\text { clicks }\end{array}$ & $\begin{array}{c}\text { The } \\
\text { number } \\
\text { of } \\
\text { mistakes }\end{array}$ & $\begin{array}{c}\text { The number } \\
\text { of } \\
\text { abandoned } \\
\text { scenario }\end{array}$ & $\begin{array}{c}\text { Satisfaction } \\
\text { scale 1 to 7 }\end{array}$ \\
\hline $\begin{array}{c}\text { Before } \\
\text { ergonomic } \\
\text { requirements }\end{array}$ & & & & & \\
\hline $\begin{array}{c}\text { After ergonomic } \\
\text { requirements }\end{array}$ & & & & & \\
\hline
\end{tabular}

\section{Conclusion}

This article proposes to integrate an ergonomic approach to improve the usability of PLM interfaces by integrating user experience through the PLM development on the agile method. The state of art demonstrates that many problems found by PLM software users as complexity or the lack of flexibility of the system could be clearly solved by including usability features. Today, PLM systems are under-utilized and too many actors of companies do not feel concerned with this kind of software. To reduce cost, improve efficiency and delays of production were the main reasons of the creation of PLM, but current software do not meet these expectations. Pernelle, Lefebvre (2006). Our model based on the agile method integrate user experience during a project development. We suppose it is improving productivity and effectiveness by improving usability. To validate our hypothesis, we establish a user tests to validate the reliability of this model and show that involving usability features and user experience tends to promote the user acceptance on the PLM interfaces. This proposition could be beneficial to increase user's satisfaction and efficiency of the companies' production.

\section{References}

1. Amann, K.: Product Lifecycle Management: Empowering the Future of Business. CIMdata, Inc. (2002)

2. Bias, R.G., Mayhew, D.J.: Cost-Justifying Usability. An Update for the Internet Age. Elsevier (2005)

3. Bossard, P.: Origines et définition de l'ingénierie concourante. In: Bossard, P., Chanchevrier, C., Leclair, P. (eds.) Ingénierie Concourante: de la Technique au Social, Economica, Paris, ch. 1, pp. 11-28 (1997)

4. Brangier, É., Hammes-Adelé, S., Bastien, J.-M.C.: Analyse critique des approches de l'acceptation des technologies: de l'utilisabilité à la symbiose humain-technologieorganisation. Revue Européenne de Psychologie Appliquée/European Review of Applied Psychology 60(2), 129-146 (2010) 
5. Donahue, G.M.: Usability and the bottom line. IEEE Software 16(1), 31-37 (2001)

6. Fadier, E.: L'intégration des facteurs humains à la conception. Phoebus, spécial sur le Facteur Humain, pp. 59-78 (1997)

7. Garetti, M., et al.: Organisational change and knowledge management in PLM implementation. International Journal of Product Lifecycle Management 1(1), 43 (2005)

8. Griffith, J.: Online transactions rise after bank redesigns for usability. The Business Journal (2002)

9. Gronier, G., Sagot, J.C.: Coopération à distance en conception de produits: analyse de l'usage d'un collecticiel. In: Battistelli, A., Depolo, M., Fraccaroli, F. (eds.) La Qualité de la vie au Travail dans les années 2000. Actes du 13ème Congrès de Psychologie du Travail et des organisations, pp. 1445-1453 (2005)

10. ISO 10303. Industrial Automation Systems and Integration - Product Data Representation and Exchange (1994)

11. ISO 13407. Human-centred design processes for interactive systems (1999)

12. ISO 9241-10. Ergonomic requirement for office work with Visual display terminal (VDTs), Part 10- Dialogue principles -First Draft International Standard (1993)

13. ISO 9241-13. Ergonomic requirements for office work with visual display terminals (VDTs) - Part 13: User guidance (1998)

14. ISO/IEC 9126. Software Product Evaluation-Quality Characteristics and Guidelines for the User, Geneva (2001)

15. ISO 9241-11. Ergonomic requirements for office work with visual display terminals (VDTs) - Part 11 Guidance on usability (1998)

16. ISO 14598. Information Technology-Software Product Evaluation, Parts, 1-5 (1999)

17. Juristo, N., Moreno, A.M., Sanchez-Segura, M.-I.: Analysing the impact of usability on software design. Journal of Systems and Software 80(9), 1506-1516 (2007)

18. Kim, G.Y., Noh, S.H., Rim, Y.H., Mun, J.H.: XML-based concurrent and integrated ergonomic analysis in PLM. The International Journal of Advanced Manufacturing Technology 39(9-10), 1045-1060 (2008)

19. Losada, B., Urretavizcaya, M., Fernández-Castro, I.: A guide to agile development of interactive software with a "User Objectives"-driven methodology. Science of Computer Programming, 1-14 (2012)

20. Maranzana, N., Segonds, F., Lesage, F., Nelson, J.: Collaborative design tools: A comparison between free software and PLM solutions in engineering education. In: Rivest, L., Bouras, A., Louhichi, B. (eds.) PLM 2012. IFIP AICT, vol. 388, pp. 547-558. Springer, Heidelberg (2012)

21. Nielsen, J., Landauer, T.K.: A mathematical model of the finding of usability problems. In: Proc. ACM INTERCHI 1993 Conf., Amsterdam, The Netherlands, April 24-29 (1993)

22. Nielsen, J.: Usability Engineering. AP Professional, Boston (1993); Perry, D., Wolf, A.: Foundations for the study of software architecture. ACM Software Engineering Notes 17(4) , 40-52

23. Pernelle, P., Lefebvre, A.: Modélisation intégrée et pérennisation des connaissances dans une approche PLM, Ingénierie de la conception et cycle de vie des produits, Traité IC2 série Productique. Hermès Science Publications (2006)

24. Preece, J., Rogers, Y., Sharp, H., Benyon, D., Holland, S., Carey, T.: Human-Computer Interaction. Addison Wesley (1994)

25. Sagot, J.C.: Ergonomie et Conception anthropocentrée. Document pour l'Habilitation a dirigé des recherches, Institut National Polytechnique de Lorraine, INPL (1999)

26. Scapin, D.L., Bastien, J.M.C.: Ergonomic criteria for evaluating the ergonomic quality of interactive systems. Behaviour \& Information Technology 16(4/5), 220-231 (1997) 
27. Seffah, A., Metzker, E.: The obstacles and myths of usability and software engineering. Communications of the ACM 47(12), 71-76 (2004)

28. Segonds, F., Iraqi-Houssaini, M., Roucoules, L., Véron, P., Aoussat, A.: The use of early design tools in engineering processes: a comparative case study. International Journal of Design and Innovation Research 5(3), 61-76 (2010)

29. Segonds, F.: Contribution to the integration of a collaborative design environment in the early stages of design. PhD thesis, Arts et Métiers ParisTech (2011)

30. Shneiderman, B.: Designing the User Interface: Strategies for Effective Human-Computer Interaction, 3rd edn. Addison Wesley, Menlo Park (1998)

31. Shneiderman, B.: Software Psychology: Human Factors in Computer and Information Systems. Winthrop Publishers, Cambridge (1980)

32. Tidwell, J.: Designing Interfaces. Patterns for Effective Interaction Design. O'Reilliy, USA (2005)

33. Trenner, L., et al.: The Politics of Usability. Springer, London (1998)

34. Welie, M.: Amsterdam Collection of Patterns in User Interface Design (2003)

35. Wognum, N., Trappey, A.: PLM challenges. Advanced Engineering Informatics 22(4), 419-420 (2008) 